\title{
Modélisation de l'impact des activités anthropiques sur les nappes phréatiques côtières Cas de Chott Maria (Sahel tunisien)
}

\author{
par R. Beni Akhy, H. Ben Dhia, A. Gamaoun \\ Ecole Nationale d'Ingénieurs de Sfax, Tunisie.
}

R. Amri

Division des Ressources en Eau du CRDA de Sousse, Tunisie.

\section{I $\square$ INTRODUCTION}

Les nappes phréatiques côtières dans le Sahel tunisien oriental sont de plus en plus influencées par les activités anthropiques diverses. Au puisage à des fins agricoles s'ajoutent les effets de l'extension urbaine. Dans certains périmètres irrigués, le pompage entraîne le risque d'intrusion des eaux marines dans ces nappes littorales. En d'autres localités, l'eau d'irrigation contribue à la recharge locale de la nappe. Ces eaux d'irrigation proviennent de nappes profondes, de barrages ou de stations d'épuration d'eaux usées. L'urbanisation est accompagnée des puits perdus qui conduisent l'eau usée d'origine domestique, touristique ou industrielle directement dans l'aquifère. La nappe phréatique urbaine de Sfax (plus au Sud), alimentée par les puits perdus au taux de 10 millions de $\mathrm{m}^{3} / \mathrm{an}$ d'eaux usées a remonté par endroits de 10 à $12 \mathrm{~m}$ et en fut bactériologiquement polluée $[1 ; 2]$.
Chott Maria, petite ville satellite de Sousse, est modeste en étendue géographique et en importance économique relativement aux grandes villes du Sahel tunisien oriental. Cependant, elle concrétise l'exemple d'une multitude de petites villes naissantes sur tout le pourtour méditerranéen méridional où la vocation agraire est mêlée à l'expansion urbaine d'une façon, malheureusement, souvent hasardeuse et fort nuisante. La nappe phréatique, dans une telle conjoncture, est à la fois la ressource en eau pour l'irrigation des cultures et le réceptacle de déchets liquides pour l'urbanisation galopante. A la pénurie périodique inhérente au climat semi-aride sud-méditerranéen, vient donc se greffer l'impact des activités anthropiques [3]. Il s'agit, dans quelques zones, de nouvelles entrées d'eau à la nappe (artificielles) telles que l'apport des puits perdus et des eaux usées recyclées ainsi que l'eau des barrages ramenée du Nord pour l'irrigation. Ailleurs, la nappe est alimentée uniquement par l'infiltration des eaux des précipitations. Cela fait autant de facteurs qui

\section{Modeling of anthropic activities impact on coastal aquifers : case of Chott Maria}

Chott-Maria's aquifer is recharged by precipitation, but also by non-treated waste-water injection through abandoned wells, partial infiltration of irrigation waters brought from the North, and treated waste waters used to irrigate gardens and golf playing-fields. Due to the urban extension, the pumping activity has moved from South to North but it has not quantitatively diminished and the risk of marine-water intrusion has not been discarded. Currently, the recharge by anthropic water injections constitute a hydraulic barrier against this marine-water intrusion. The mathematical modeling showed that the generalisation of the collective drain-network, by 2001, should be preceded by a redistribution, in space and time, of the pumping areas and rates. Otherwise, a marine-water intrusion with a width of $3 \mathrm{~km}$ would affect the northern sea-coast since 2006. 


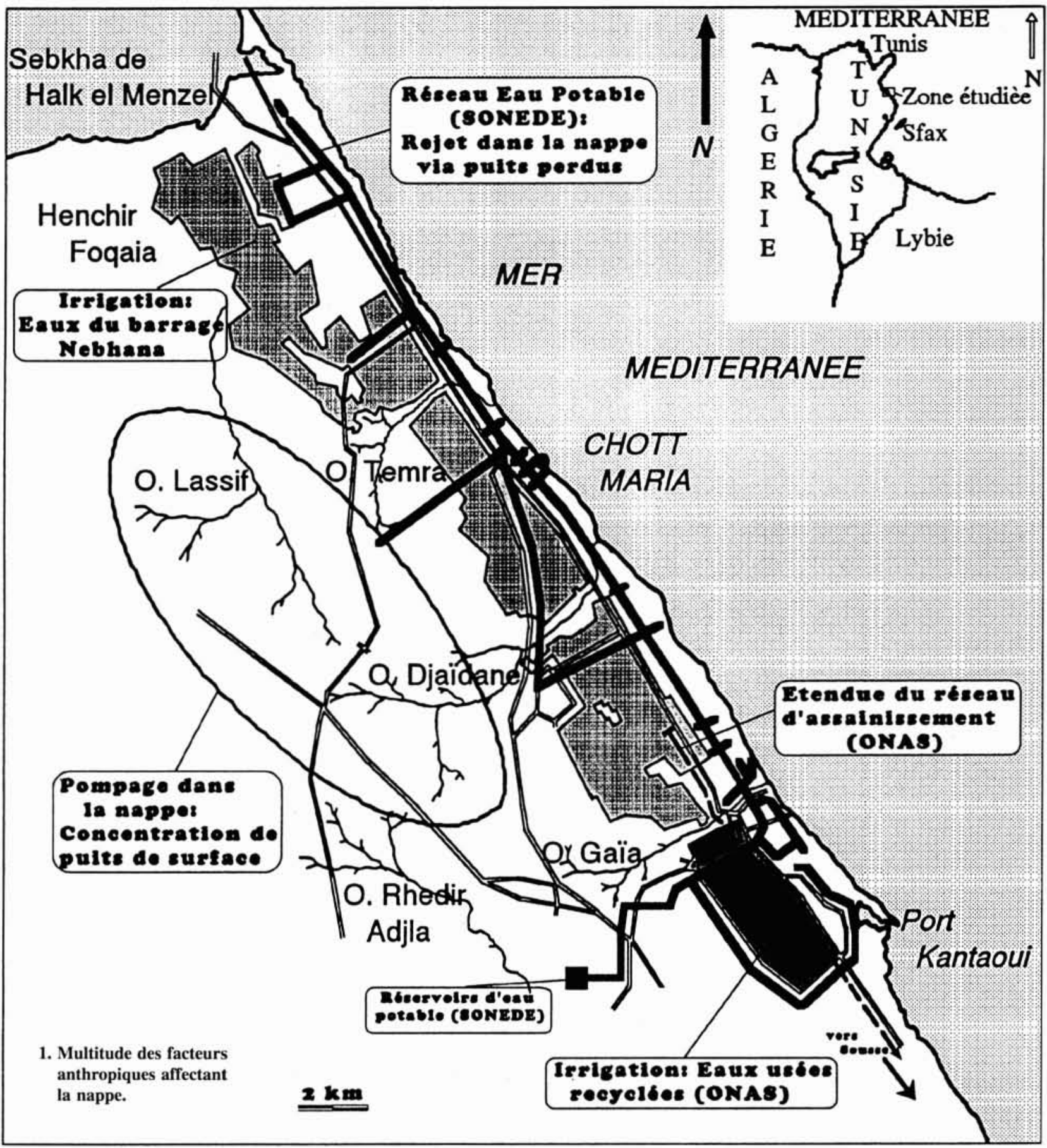

rendent le bilan en eau de la nappe très complexe et son équilibre hydrodynamique fragile et surtout très aléatoire. La pollution organique et éventuellement chimique seraient considérables mais nous nous intéressons ici à l'aspect hydrodynamique de la nappe que nous traitons à l'aide de l'outil numérique, seule approche susceptible d'aborder quantitativement le bilan en eau et de prévoir, par les simulations prévisionnelles, les futurs risques et menaces.

La région de Chott Maria (4000 habitants repartis sur 1300 familles) est située dans le centre du Sahel tunisien méditerranéen septentrional, à $7 \mathrm{~km}$ au nord de la ville de Sousse. Elle a pour principales productions l'olivier (2000 ha) et les cultures maraîchères (576 ha dont 40 ha sous serre) qui produisent des primeurs pendant toute l'année. Les emplois relevant du domaine agricole sont occasionnels et les services et le commerce se développent de part et d'autre de la route touristique. Les résidences hôtelières et les résidences se concentrent au sud de la région, le nord de la station de plaisance étant intégré au port El Kantaoui. Le tourisme commence à l'emporter sur l'activité agricole en terme de main d'œuvre.

La région peut être partagée en quatre secteurs (fig.1) :

- le noyau urbain qui constitue la ville de Chott Maria ;

- la zone d'habitation spontanée, en groupuscules de demeures qui s'étendent en "tâche d'huile" et d'une manière anarchique le long de la route et au détriment des terres cultivées (Dar Ben Zina et $\mathrm{Al}$ Homran par exemple);

- la zone hôtelière qui s'étale le long du rivage sud et le terrain de golf d'El Kantaoui; 
- les Périmètres Publics Irrigués (PPI).

La précipitation annuelle moyenne durant les 21 dernières années (de 1975 à 1995) est égale à $382 \mathrm{~mm}$ alors qu'elle était de $350 \mathrm{~mm}$ pour la période de 1901/1970. Le climat est de type semi-aride et la grande irrégularité du régime des précipitations ainsi que les sécheresses périodiques en sont le signe le plus notable. La température moyenne est de $19^{\circ} \mathrm{C}$.

L'amplitude thermique annuelle, influencée par l'effet modérateur de la mer, est de $15^{\circ} \mathrm{C}\left(18,5^{\circ} \mathrm{C}\right.$ à Kairouan). L'évapotranspiration réelle, évaluée par la méthode de Turc, est de $377 \mathrm{~mm}$. L'évapotranspiration potentielle (ETP) moyenne mensuelle calculée pour la période allant de 1981 jusqu'à 1995 est de $77 \mathrm{~mm} / \mathrm{mois}$. L'évaporation moyenne mensuelle Piche est de $29 \mathrm{~mm}$.

\section{II $\square$ BILAN EN EAU}

La nappe phréatique côtière de Chott Maria est logée dans des couches mio-pliocènes faites d'alternance de bancs de sable avec des marnes franches ou sableuses. Elle s'écoule vers la mer à l'Est et vers la Sebkha de Halk El Menzel au Nord. Ses ressources exploitables (correspondant grossièrement aux réserves régulatrices), estimées à partir du taux d'alimentation, sont de $0,86 \mathrm{Mm}^{3} /$ an. Le renouvellement des mensuellement (où l'on calcule la recharge de la nappe à partir de la remontée de son niveau induite par une averse). Ainsi, sur la superficie de $45 \mathrm{~km}^{2}$, et pour une pluviométrie annuelle moyenne de $382 \mathrm{~mm} / \mathrm{an}$, l'apport des eaux météoriques à l'aquifère est voisin de $860000 \mathrm{~m}^{3} / \mathrm{an}$. C'est une estimation de l'apport total des eaux météoriques à la nappe, y compris au niveau des lits des Oueds Djä̈dane, Temra, Lassif, Rhedir Adjela et Gaïa. En effet, ces Oueds sont très courts ( 2 à $3,5 \mathrm{~km})$, ruissellent très rarement à l'occasion des crues exceptionnelles (une durée de crue de 10 heures par an a été estimée au Cap-Bon, plus au Nord, par la DRE) et aucun cours d'eau n'établit une liaison hydraulique directe avec la nappe même à l'embouchure. De plus, le dénivellement topographique des lits de ces Oueds est faible et progressif (moins que $10 \mathrm{~m}$ ) et ils sont cultivés, habités et parsemés de puits. Les sols de faciès sableux perméable dominent toute la région étudiée et permettent une recharge sensiblement homogène comme le montrent les profils de remontée de la nappe après les précipitations (à la différence d'autres aquifères phréatiques du Sahel comme la nappe phréatique de Sfax où les apports des Oueds Maou, Aguareb et el-Aouabed représentent $25 \%$ de la recharge totale).

Cependant, la pluviométrie annuelle oscille amplement autour de la moyenne annuelle et son apport à la nappe demeure très variable.

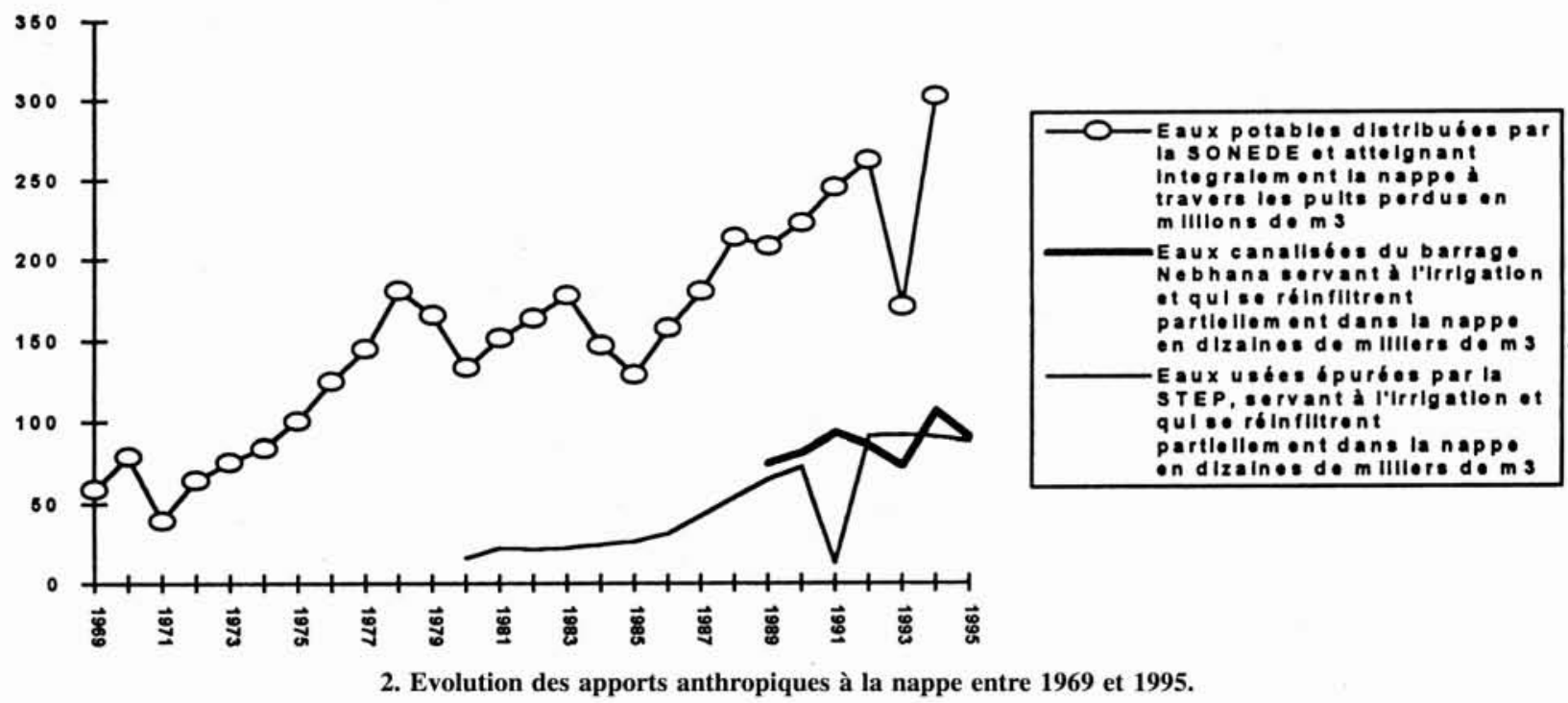

réserves régulatrices est tributaire des conditions météoriques et l'exploitation actuelle est légèrement supérieure à la moitié de la ressource dynamique annuelle avec $0,46 \mathrm{Mm}^{3} / \mathrm{an}[5]$.

Le chimisme de l'eau de la nappe est à dominante chloruré sodique et la transmissivité avoisine les $2 \cdot 10^{-4} \mathrm{~m}^{2} / \mathrm{s}$. La région étant de plus en plus envahie par l'extension urbaine et l'activité hôtelière et touristique, la nappe est très sollicitée par l'Homme pour des usages très variés (fig. 1). La recharge s'accomplit principalement par les eaux de pluie et à un degré moindre, à partir des rejets des eaux usées domestiques dans les puits perdus et de la réinfiltration d'une partie des eaux d'irrigation. La décharge se fait principalement par le pompage à des fins agricoles.

\subsection{Recharge par l'infiltration des eaux de précipitation}

L'apport à la nappe par les eaux des précipitation a été estimé sur la base d'un coefficient d'infiltration de $5 \%$ déterminé par la méthode de Kamenski appliquée à des triplets de puits alignés sur une ligne d'écoulement et suivis

\subsection{Recharge par les puits perdus}

Les eaux potables distribuées par la Société Nationale d'Exploitation et de Distribution des Eaux (SONEDE) finissent dans les puits perdus après leur usage domestique compte tenu de l'absence totale de réseau d'assainissement sur toute l'étendue de la nappe, à l'exception de la zone hôtelière branchée au réseau de la Station d'Epuration (STEP) de Sousse-Nord. Le réseau SONEDE (eau potable) de la commune Chott Maria est indépendant de celui de la zone hôtelière. Les eaux potables rejetées dans les puits perdus qui communiquent directement avec la nappe finissent par s'y infiltrer quasi-totalement. Pour notre calcul, nous pouvons donc estimer que les eaux distribuées par la SONEDE dans cette région dépourvue de réseau d'égouts est un apport régulier à la nappe (fig. 2). Les volumes annuels d'eau potable consommés depuis 1969 s'échelonnent entre $60.10^{3}$ et $300.10^{3} \mathrm{~m}^{3} / \mathrm{an}$ avec une moyenne annuelle de $200.10^{3} \mathrm{~m}^{3}$ et une évolution annuelle de $9 \%$. Cela fait alors un apport annuel moyen à la nappe de $171,7.10^{3} \mathrm{~m}^{3} /$ an (tableau 3 ). 


\begin{tabular}{|l|c|c|c|c|c|c|c|c|}
\hline & 1989 & 1990 & 1991 & 1992 & 1993 & 1994 & 1995 & Moyennes \\
\hline $\begin{array}{l}\text { Irrigation par l'eau } \\
\text { du barrage (Périmètres } \\
\text { Publiques Irrigués)[6] }\end{array}$ & 745,1 & 809,8 & 926 & 851,6 & 726,3 & 1063,6 & 905,2 & 861 \\
\hline Recharge de la nappe & 76 & 140,8 & 257 & 182,6 & 57,3 & 394,6 & 236,2 & 192 \\
& $10,1 \%$ & $17,4 \%$ & $27,8 \%$ & $21,4 \%$ & $7,9 \%$ & $37,1 \%$ & $29,1 \%$ & $22,3 \%$ \\
\hline $\begin{array}{l}\text { Irrigation par l'eau traitée } \\
\text { (terrain de golf) [6] }\end{array}$ & 650,1 & 720,8 & 728,8 & 908,8 & 919,8 & 904,2 & 879,8 & 781,5 \\
\hline Recharge de la nappe & 210,1 & 280,8 & 288,8 & 468,8 & 479,8 & 464,2 & 439,8 & 341,5 \\
& $32,3 \%$ & $38,9 \%$ & $38,5 \%$ & $51,6 \%$ & $52,2 \%$ & $51,3 \%$ & $49,9 \%$ & $43,7 \%$ \\
\hline Irrigation totale & 1395,2 & 1530,6 & 1654,8 & 1760,4 & 1646,1 & 1967,8 & 1785 & 1642,5 \\
\hline Recharge totale & 286,1 & 421,6 & 545,8 & 651,4 & 537,1 & 858,8 & 700,4 & 533,3 \\
& $20,5 \%$ & $27,5 \%$ & $32,9 \%$ & $37 \%$ & $32,6 \%$ & $43,6 \%$ & $39,2 \%$ & $32,5 \%$ \\
\hline
\end{tabular}

Tableau 1. - Alimentation de la nappe par l'infiltration d'une partie des eaux d'irrigation (milliers de $\mathrm{m}^{3} / a n$ ).

\subsection{Recharge par l'infiltration d'une partie des eaux d'irrigation}

L'irrigation concerne deux sites :

(i) le Périmètre Public Irrigué de Chott Maria qui s'étend sur une superficie de 576 ha dont environ $6 \%$ sous serre (cultures potagères). Ce périmètre se situe à $8 \mathrm{~km}$ à l'Ouest de la route touristique. Il s'approvisionne en eau à partir du barrage de Sidi Messaoud (Nebhana) et de quatre forages profonds. Le barrage de Sidi Messaoud, prenant place sur Oued Nebhana, $47 \mathrm{~km}$ au nord-ouest de Kairouan, fut construit entre 1962 et 1966 et a commencé à desservir d'une manière effective la région depuis 1972. Les eaux provenant du barrage sont stockées dans un bassin de collecte à l'ouest de Chott Maria avant d'être distribuées dans les périmètres irrigués. Des pertes d'environ $30 \%$ affectent le réseau à l'intérieur de ces périmètres [6].

(ii) le terrain de golf d'El Kantaoui et les jardins de quelques hôtels, couvrant ensemble une superficie avoisinant les 200 ha, qui sont irrigués par $20 \%$ du volume total des eaux usées traitées à la station d'épuration de Sousse-Nord pendant six mois de l'année [7].

Les pertes du réseau, ainsi que l'irrigation des terrains de golf contribuent à la recharge pendant toute l'année. La composante ruissellement étant négligeable, l'eau d'irrigation non utilisée par les plantes et qui n'est pas évaporée alimente la nappe. Plusieurs travaux visent à l'évaluation expérimentale des besoins en eau des plantes. Schoeller [8] ainsi que McDonald [9], pour les mêmes latitudes que notre zone d'étude, citent des chiffres proches de $1300 \mathrm{~m}^{3} / \mathrm{ha} / \mathrm{an}$ pour les cultures maraîchères et $2200 \mathrm{~m}^{3} / \mathrm{ha} /$ an pour le gazon du terrain de golf. Ces besoins en eau englobent l'évapotranspiration. Pour estimer la fraction d'eau d'irrigation qui alimente la nappe, nous avons calculé la différence entre les volumes d'eau lâchés dans les périmètres irrigués et la quantité d'eau utilisée réellement par les cultures. La nappe se rechargerait ainsi, en moyenne, de $192000 \mathrm{~m}^{3} / \mathrm{an}$ au niveau des Périmètres Publiques Irrigués et de $341500 \mathrm{~m}^{3} / \mathrm{an}$ au niveau du terrain de golf d'El Kantaoui (tableaux 1 et 3 et fig. 2). Cela correspond à $22,3 \%$ de l'eau d'irrigation au niveau des périmètres de maraîchage et à 43,7 \% (en moyenne) au niveau du terrain de golf. En somme, sur un volume total moyen d'eau d'irrigation de 1,64 million de $\mathrm{m}^{3} / \mathrm{an}, 0,53$ millions $\mathrm{de}^{3} / \mathrm{an}$ alimentent la nappe, soit $32 \%$.

\subsection{Décharge}

Le volume annuel moyen des prélèvements par pompage est estimé à $330.10^{3} \mathrm{~m}^{3}$ en 1984 et à $580.10^{3} \mathrm{~m}^{3}$ en 1991 [10]. Le nombre de puits, équipés en majorité de groupes moto-pompes électriques ou diesel, a évolué de 191 en 1984 à 207 en 1991 (tableau 2). Environ 70 points d'eau (soit le tiers du nombre total) sont délaissés soit à cause de la médiocrité de la qualité de l'eau (parcelle nord avoisinant la Sebkha et zone côtière méridionale), soit en raison de l'extension du réseau d'eau potable. Ces puits abandonnés ont été alors transformés en puits perdus dans un rayon de $1,5 \mathrm{~km}$ autour du village de Chott Maria.

\begin{tabular}{|c|c|c|c|c|c|}
\hline Puits & $\begin{array}{c}\text { électro } \\
\text { moto- } \\
\text { pompe }\end{array}$ & $\begin{array}{c}\text { diesel } \\
\text { moto- } \\
\text { pompe }\end{array}$ & $\begin{array}{c}\text { aban- } \\
\text { donnés }\end{array}$ & Total & $\begin{array}{c}\text { pompage } \\
\text { en } 10^{3} \\
\mathrm{~m}^{3} / \mathrm{an}\end{array}$ \\
\hline $\begin{array}{c}\text { En } \\
1984\end{array}$ & 91 & 20 & 2 & 191 & 330 \\
\hline $\begin{array}{c}\text { En } \\
1991\end{array}$ & 113 & 23 & 3 & 210 & 580 \\
\hline
\end{tabular}

Tableau 2. - Exploitation de la nappe [10].

La moyenne du pompage durant les douze dernières années (1984 à 1996) est de $456,5 \cdot 10^{3} \mathrm{~m}^{3} / \mathrm{an}$. Le pompage dans la nappe est concentré dans la région de Oued Lassif et serait à l'origine du front de salinisation par les eaux marines plus en aval de l'écoulement.

L'écoulement des eaux souterraines vers la mer et la Sebkha de Halk El Menzel est estimé, à l'aide de la loi de Darcy, sur un front d'écoulement de $13 \mathrm{~km}$, avec un gradient hydraulique moyen de $5 \%$ et une transmissivité de $2.10^{-4} \mathrm{~m}^{2} / \mathrm{s}$, à $417,8.10^{3} \mathrm{~m}^{3} /$ an.

Le bilan en eau (tableau 3 ) résulte d'un calcul, certes, simplificateur et comporte plusieurs estimations. Néanmoins, une telle approche est nécessaire pour comprendre le fonctionnement complexe de la nappe. Ce bilan met en exergue une recharge largement influencée par l'apport de l'infiltration des eaux de pluie. Toutefois, la répartition spatiale des aires d'influence des différents facteurs de ce bilan est hétérogène. Ainsi, en dépit de la recharge considérable par les eaux d'irrigation et les puits perdus, la nappe est menacée localement d'une intrusion d'eau marine.

\begin{tabular}{|l|l|}
\hline Infiltration des eaux de pluie : 860 & $\begin{array}{l}\text { Ecoulement occulte } \\
\text { aux exutoires : } 418\end{array}$ \\
\hline $\begin{array}{l}\text { Puits perdus et fosses septiques : } \\
171,7\end{array}$ & $\begin{array}{l}\text { Prélèvements : } \\
456,5\end{array}$ \\
\hline $\begin{array}{l}\text { Réinfiltration des eaux } \\
\text { d'irrigations : } 533,3\end{array}$ & \\
\hline Total: 1565 & Total: 874,5 \\
\hline
\end{tabular}

Tableau 3: Bilan hydrique de la nappe $\left(10^{3} \mathrm{~m}^{3} / \mathrm{an}\right)$. 


\section{III $\square$ HYDRODYNAMISME}

L'état de la nappe est suivi au niveau de puits sélectionnés où la Division des Ressources en Eau (DRE) de Sousse effectue des mesures de profondeur du plan d'eau et de salinité deux fois par an. Trois inventaires généralisés ont été effectués respectivement en avril 1969, septembre 1984 et septembre 1991 (mesures de la piézométrie et de la salinité totale au niveau de 25 puits). Notre campagne de mesures réalisée en avril 1996 (une trentaine de puits parmi 200 inventoriés) s'est intéressée, en plus du niveau de la nappe et de sa salinité, au nivellement topographique précis des points d'eau. Cela nous a servi à la correction des données relatives aux anciens inventaires, la précision des données étant un atout considérable pour la qualité du modèle mathématique.

La profondeur des eaux de la nappe s'échelonne entre $2 \mathrm{~m}$ au voisinage de la côte et légèrement plus de $50 \mathrm{~m}$ en amont. Sur les cartes d'isoprofondeur de 1984 et de 1991, une concavité des courbes isobathes orientée SW-NE souligne bien la dépression de Oued Lassif. En Avril 1996, en revanche, la profondeur de la nappe tend plutôt à être homogène. Les pluies exceptionnelles de l'année 1996 semblent donc avoir régularisé la profondeur des eaux. En septembre 1969 [11], l'écoulement est uniforme de direction W-E au Sud et SW-NE au Nord et au centre et le gradient hydraulique est faible de l'ordre de $3 \%$ (fig. $3 a$ ). A partir de septembre 1984 (fig. $3 b$ ), et contrairement à la partie sud, l'écoulement se perturbe dans la moitié

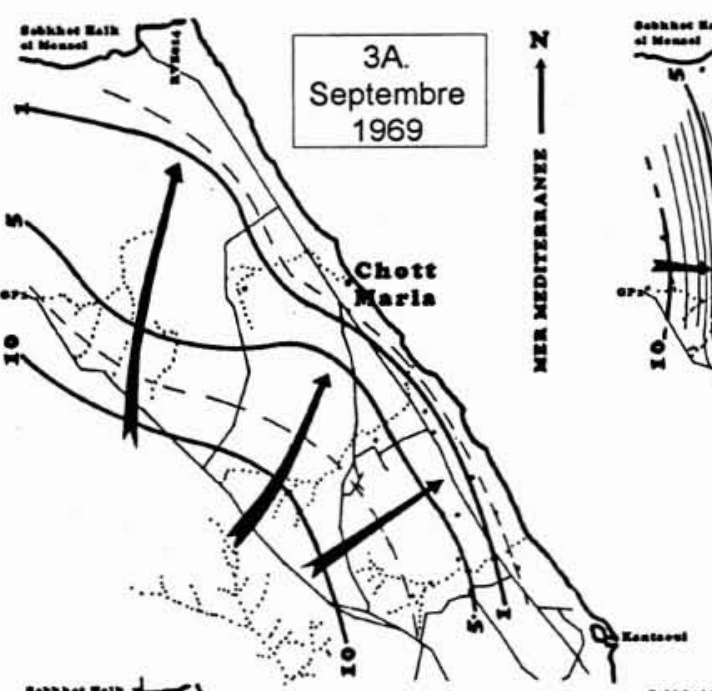

Oued Nebhana et parallèlement à la route RVE 814 que longe le réseau de desserte en eau potable alimentant totalement la nappe à travers les puits perdus. L'infiltration de ces eaux d'irrigation et celle des eaux usées domestiques sont autant de facteurs qui auraient contribué à l'érection de cette structure en dôme piézométrique. Cette dernière n'apparaît pas sur la carte d'avril 1996, cette année étant de pluviométrie exceptionnellement excédentaire et les mesures ayant été nord de la nappe où se crée et persiste (septembre 1984, fig. $3 b$ et septembre 1991, fig. $3 c$ ), jusqu'à notre dernier inventaire en avril 1996 (fig. 3d), une dépression piézométrique au niveau de la vallée de Oued Lassif. Des écoulements inverses (du NE vers le SW) apparaissent dans cette aire de convergence de filets liquides où le pompage dans la nappe ( $7000 \mathrm{~m}^{3} /$ puits/an) se trouve favorisé par :

(i) la migration de l'activité agricole du Sud (où nombre de puits furent abandonnés) et sa concentration au Nord de la ville;

(ii) la topographie qui atténue la profondeur de la nappe allant, selon les mesures d'avril 1996, de 20 à 45 m;

(iii) la bonne salinité relative des eaux qui n'a jamais dépassé les $3 \mathrm{~g} / \mathrm{l}$.

Comme le montrent les cartes de 1984 et 1991 (fig. 3), une structure en dôme piézométrique s'interpose entre la vallée de Oued Lassif et la côte $[12,8]$ à l'emplacement des périmètres irrigués par les eaux ramenées du barrage de faites en pleine saison pluvieuse. Dans la partie de la nappe située au Sud de Chott Maria, l'écoulement reste régulier du $\mathrm{SE}$ vers le NW avec un gradient hydraulique élevé de 5 à $8 \%$ [13].

\section{IV ■ SALINITÉ}

En dépouillant les résultats des suivis annuels et continus de quelques puits, effectués par la DRE de Sousse depuis 1972 jusqu'à 1993, nous constatons que la salinité dans la zone au Sud de la Sebkha Halk El Menzel a montré une tendance à la stabilité (entre 2,3 et $2,6 \mathrm{~g} / \mathrm{l}$ de sels totaux) pendant la période comprise entre septembre 1972 et septembre 1987 suivie d'une augmentation relative ( 3 à $3,4 \mathrm{~g} / \mathrm{l}$ ) qui persiste jusqu'à mai 1993.

Le secteur côtier, du fait de sa proximité du rivage, est d'une salinité très variable (elle oscille entre $8 \mathrm{~g} / \mathrm{l}$ et $3 \mathrm{~g} / \mathrm{l}$ ). La succession de trois années pluvieuses (1974/1975 à 
1976/1977) a dilué la salinité dans certains puits de $7,5 \mathrm{~g} / \mathrm{l}$ en septembre 1975 à $3,65 \mathrm{~g} / \mathrm{l}$ en septembre 1977. D'autre part l'année sèche 1977/1978 $(248 \mathrm{~mm})$ a engendré, dans ces mêmes puits, un pic de salinité de 5,3 g/l en octobre 1978, alors qu'elle était de $4 \mathrm{~g} / \mathrm{l}$ en septembre 1977. Alors qu'il se situe à $1 \mathrm{~km}$ de la mer, le secteur central irrigué par les eaux du barrage de Nebhana, est d'une salinité inférieure à $3 \mathrm{~g} / \mathrm{l}$ avec parfois des pics de salinité relativement élevés $(3,65 \mathrm{~g} / 1$ en septembre $1980,3,2 \mathrm{~g} / \mathrm{l}$ en septembre 1985 et $3,5 \mathrm{~g} / \mathrm{l}$ en septembre 1989). Il s'agirait de l'effet des basses pluviométries qui sont $248,2 \mathrm{~mm}$ en $1977 / 78, \quad 234,6 \mathrm{~mm}$ en $1983 / 84$ et $191,3 \mathrm{~mm}$ en $1987 / 88$. On note ainsi la relative rapidité de réponse de la nappe, en terme de salinité, au déficit pluvial. A l'opposé, certains puits dans ce secteur ont des eaux de salinité élevée, qui oscille entre $4,5 \mathrm{~g} / \mathrm{l}$ et $6 \mathrm{~g} / \mathrm{l}$ avec une tendance à se stabiliser à $5 \mathrm{~g} / \mathrm{l}$. Le secteur amont de la nappe se caractérise par une salinité réduite et faiblement variable.

La carte de salinité de la nappe en septembre 1984 montre que la charge des eaux dépassait les $5 \mathrm{~g} / \mathrm{l}$ (localement $8 \mathrm{~g} / \mathrm{l}$ ) le long du rivage méridional. Une zone restreinte de salinité comprise entre 4 et $7 \mathrm{~g} / \mathrm{l}$ se dessinait au Sud de Henchir El Foqaïa tandis qu'une aire de faible et moyenne salinité (moins que $3 \mathrm{~g} / \mathrm{l}$ ) se localisait au Sud et au Sud-Ouest et dans la partie centrale. La charge saline élevée à l'extrême Nord au voisinage de la Sebkha confinée paraît naturelle
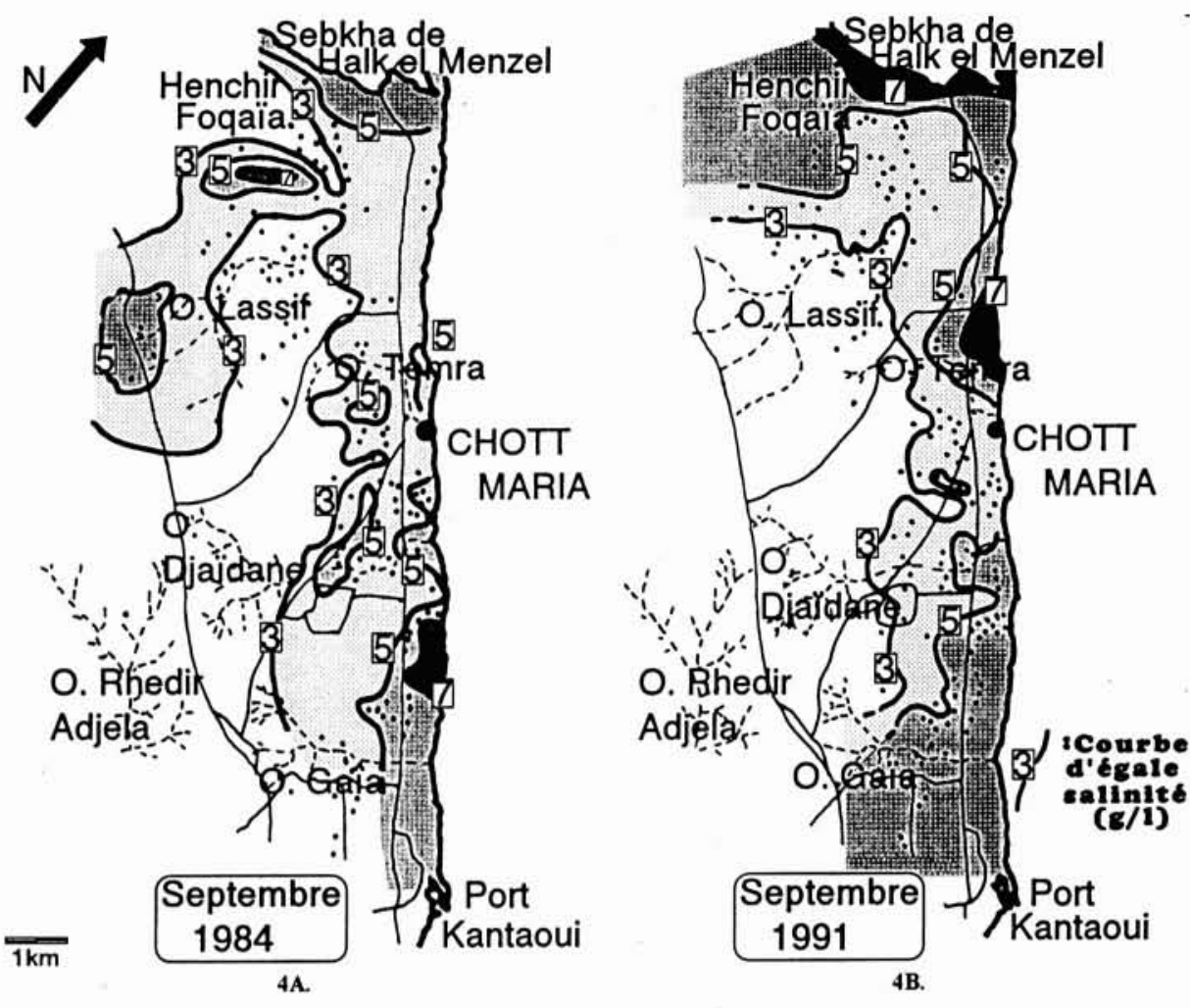

4. Evolution de la salinité des eaux.

alors qu'au Sud du village, la salinité qui dépasse les $7 \mathrm{~g} / \mathrm{l}$ semble être liée à l'activité de pompage par la forte densité de puits de surface $[14,15]$. Suite à la reconversion de cette zone aux activités touristiques, plusieurs puits ont été délaissés et le puisage dans la nappe s'est déplacé vers le Nord du village.

A partir de 1991 (carte de la figure 4B.), le littoral nord commence, à son tour, à être dominé par des salinités supérieures à $5 \mathrm{~g} / \mathrm{l}$. Un front de salinité de $7 \mathrm{~g} / \mathrm{l}$ apparaît près de l'embouchure de Oued Temra, en aval de la région Oued Lassif où la création de puits et l'activité de pompage se sont renforcés.

Ce front de salinité persiste actuellement et menace de s'élargir pour créer une aire potentielle d'avancée du biseau salé. Notons aussi la nette diminution de la salinité en liaison avec les pluies de la fin 1995 et du début 1996 le long des côtes, particulièrement celles qui s'ouvrent sur la Sebkha et au niveau de l'embouchure de Oued Jaïdane. Cette baisse de la salinité, due à l'infiltration des eaux de précipitation, ne toucherait toutefois que la tranche supérieure des eaux de la nappe. De plus, l'apport à la nappe à partir de l'infiltration des eaux d'irrigation (salinité inférieure à $2 \mathrm{~g} / \mathrm{l}$ ) aurait permis de maintenir une salinité acceptable. Ce phénomène

5. Maillage et conditions aux limites du modèle et paramètres hydrauliques retenus après calage. 
s'amplifie lors des périodes peu pluvieuses et touche essentiellement le secteur des périmètres publiques irrigués, situé sur la bordure ouest de la route touristique.

\section{V — MODÉLISATION}

Il s'agit d'une simulation numérique monocouche sur le programme MULTIC [16]. Le modèle englobe 179 mailles carrées régulières de $500 \mathrm{~m}$ de côté (fig. 5). Le programme intègre l'équation aux dérivées partielles décrivant l'écoulement bidimensionnel par la méthode des différences finies appliquées au réseau de mailles carrées régulières. L'approximation de la dérivée partielle dans le temps est exécutée au moyen d'un schéma implicite pur. Les systèmes d'équations linéaires sont résolus par la méthode de GaussSeidel avec surrelaxation par point. Un potentiel imposé nul a été affecté aux mailles qui côtoient le rivage méditerranéen et la Sebkha (fig. a).

\subsection{Simulation de l'écoulement en régime permanent}

L'état de la piézométrie en septembre 1969, matérialisant la situation d'un écoulement pseudo-permanent de la nappe, a été fixé comme référence au modèle. Au demeurant, on a attribué à toutes les mailles une transmissivité égale à $2.10^{-4}$ $\mathrm{m}^{2} / \mathrm{s}$, valeur induite de deux essais de pompages qui ont eu lieu l'un au Nord du secteur (à Henchir El Foqaïa) et l'autre à son extrémité sud. L'apport hydrique à la nappe à partir de la pluie (y compris au niveau des lits d'Oueds) prime sur les débits imposés entrants avec $859.10^{3} \mathrm{~m}^{3} /$ an pour une précipitation de $382 \mathrm{~mm} / \mathrm{an}$ (relativement aux autres entrées artificielles). Le nombre des puits de surface qui sollicitaient la nappe en 1969 était modeste (12 puits) ainsi que le volume annuel pompé $\left(1,5.10^{3} \mathrm{~m}^{3} /\right.$ puits/an) qui se répartit ça et là sur le rivage central et méridional. Le calage du modèle en régime permanent a porté sur la répartition des transmissivités et le contrôle de la validité de ce calage s'est fait sur la totalité des mailles (fig. $5 a)$. L'écart de calage maximal entre les charges hydrauliques calculées et celles mesurées atteint $1 \mathrm{~m}$ et apparaît en amont de la nappe où la piézométrie est de $20 \mathrm{~m}$. Les transmissivités retenues après le calage du modèle en régime permanent vont de 2 à $40.10^{-4} \mathrm{~m}^{2} / \mathrm{s}$. En concordance avec la nature alluviale de l'aquifêre, les transmissivités fixées par calage sont élevées au niveau des lits de Oued Lassif $\left(30.10^{-4} \mathrm{~m}^{2} / \mathrm{s}\right)$ et de Oued Jaïdane $\left(25\right.$ à $\left.40.10^{-4} \mathrm{~m}^{2} / \mathrm{s}\right)$.

\section{- 5.2 Simulation de l'écoulement en régime transitoire}

La simulation en régime transitoire consiste à reproduire le fonctionnement hydrodynamique de la nappe pendant les 27 dernières années (1969/1996). Compte tenu des cartes piézométriques dont on dispose $(1969,1984,1991$ et 1996), on s'est fixé trois phases de simulation en régime transitoire : une première phase de 1969 à 1984, une deuxième de 1984 à 1991 et une troisième de 1991 à 1996 . La nappe est moyennement sollicitée le long du rivage central et sud ainsi qu'au niveau de Henchir El Foqaïa, et grandement exploitée dans la région de Oued Lassif. Lors de chaque phase de simulation, on a adopté une valeur moyenne des débits entrants en tenant en compte de :

(i) l'infiltration des eaux de pluie successivement sur les cycles suivants : 1969/1984, 1984/1991 et $1991 / 1996$ qui est respectivement de 738900,844400 et $1600200 \mathrm{~m}^{3} / \mathrm{an}$;

(ii) les quantité moyennes d'eaux usées domestiques parvenant à recharger la nappe de Chott Maria qui sont de $118300 \mathrm{~m}^{3} /$ an entre 1969 et $1984,187,700 \mathrm{~m}^{3} / \mathrm{an}$ entre 1985 et 1991 et $269600 \mathrm{~m}^{3} /$ an entre 1992 et 1995 . Ces rejets concernent les lieux desservis par la SONEDE et dépourvus d'un réseau d'égouts;

(iii) l'apport à la nappe par l'infiltration des eaux d'irrigation du barrage Nebhana au niveau des périmètres irrigués de Chott Maria, introduit depuis la deuxième phase où la moyenne en est de $41000 \mathrm{~m}^{3} / \mathrm{an}$ et qui contribue à l'alimentation de l'aquifère en moyenne avec $44000 \mathrm{~m}^{3} / \mathrm{an}$ entre 1992 et 1995 ;

(iv) l'apport à la nappe à partir des eaux usées recyclées issues de la station d'épuration de Sousse-Nord et utilisées pour l'arrosage (extrémité sud de la région) qui est de $14900 \mathrm{~m}^{3} /$ an en première phase, $31000 \mathrm{~m}^{3} /$ an en deuxième, et $63200 \mathrm{~m}^{3} / \mathrm{an}$ en troisième phase.
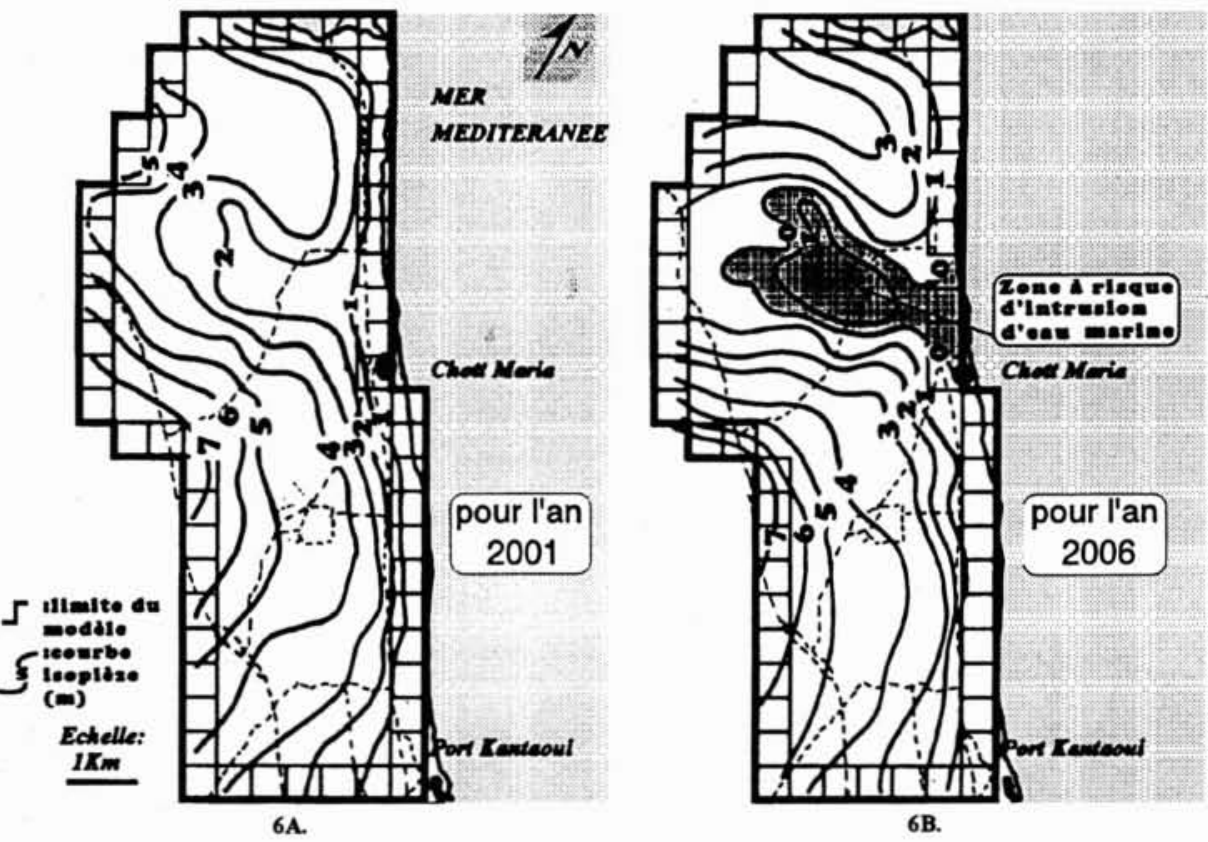

6B.

Cartes piézométriques calculées pour 2001 et 2006 dans les conditions de simulation et risque prévu de dégradation de la qualité des eaux.

Le calage en régime d'écoulement transitoire consiste à vérifier le fonctionnement hydraulique du modèle par phase en agissant sur les paramètres hydrauliques, notamment le coefficient d'emmagasinage [17]. Chaque simulation en régime transitoire restitue des cartes piézométriques calculées pour les années 1984, 1991 et 1996 qui sont confrontées aux cartes réelles. Les coefficients d'emmagasinage retenus après calage s'échelonnent entre $10^{-3}$ et $4 \cdot 10^{-2}$ (fig. $5 b$ ). La répartition de ces coefficients qu'a nécessité le calage du modèle révèle des valeurs élevées de ce paramètre dans la moitié nord-ouest de la nappe ainsi que dans la zone de Swani Ladhari. Cela correspond aux lits de Oued Temra (30) et de Oued Lassif ( 30 à 40 ) où la nappe est très productive et sollicitée. Par ailleurs, des valeurs faibles couvrent toute la bande côtière ainsi que la zone méridionale et sud-ouest (fig. $5 b$ ). 


\section{- 5.3 simulation prévisionnelle}

Le calcul du bilan hydrique ainsi que l'étude hydrodynamique de la nappe ont confirmé, outre l'influence des différents apports de nature anthropique, la relation intime : pluie-comportement quantitatif et qualitatif de la nappe.

Nous présentons la simulation du scénario le plus vraisemblable visant l'an 2001 et l'an 2006 lorsqu'on tient compte des facteurs démographiques, socio-économiques et naturels dont dépend l'équilibre hydrodynamique de la nappe. Dans l'horizon 2001, nous simulons la succession de cinq années sèches avec une moyenne annuelle de $180 \mathrm{~mm} / \mathrm{an}$. Par ailleurs, cette simulation suppose que l'apport à la nappe par les rejets d'eau usée domestique suivrait une croissance linéaire annuelle de l'ordre de $9 \%$ (l'évolution annuelle de la consommation d'eau potable de Chott Maria pendant la période 1969-1994). Les eaux usées continueraient à atteindre la nappe puisque, selon les responsables de l'Office National de l'Assainissement Sanitaire (ONAS), aucun projet d'assainissement de la région n'est envisagé avant l'an 2001 (hypothèse simulée). Le taux de raccordement actuel à la STEP Sousse-Nord est estimé à $80 \%$. D'ici l'an 2001, l'évolution du volume d'eaux usées accédant à cette station d'épuration ne prévoit qu'une croissance de l'ordre de $5 \%$ (selon les responsables de l'ONAS de Hammam Sousse). L'état d'exploitation simulée dans cet horizon est renforcée par la création, dans la région de Oued Lassif, de 45 nouveaux puits par an pompés à raison de $4000 \mathrm{~m}^{3} /$ an/puits. A l'horizon 2006 nous simulons :

(i) une pluviométrie annuelle égale à la moyenne des 21 dernières années (1975-1995) qui est de $382 \mathrm{~mm} / \mathrm{an}$;

(ii) la création d'une station d'épuration à l'extrémité nord de la région (assainir conjointement le village de Chott Maria et la ville de Hergla);

(iii) l'élimination des rejets d'eau usée domestique dans la nappe suite à l'assainissement total de la commune.

Les cartes piézométriques calculées dans ces conditions de simulation (d'ailleurs les plus vraisemblables), prévoient, à l'horizon 2001 (fig. 6a), le retrait du front de hauteur piézométrique $2 \mathrm{~m}$ plus à l'Est créant une zone de faiblesse hydrodynamique de la nappe envers la pression de l'eau marine immédiatement au Nord de la ville. Cette aire évoluerait franchement, d'ici l'an 2006 (fig. 6b), vers une dépression d'altitude plus basse que le niveau marin. Cela signifie l'intrusion nette des eaux marines salées sur un front de $3 \mathrm{~km}$ à l'Est du rivage. Cette zone à risque apparaît directement en aval du périmètre de Oued Lassif où l'on pratique encore le pompage dans la nappe et où un équilibre relatif se maintient actuellement grâce aux apports de nature anthropique. Ainsi, l'apport compensateur des rejets domestiques dans les puits perdus devant disparaître d'ici l'an 2001, la nappe perdrait son équilibre hydrodynamique fragile si le rythme actuel de création de puits se maintenait. Le modèle montre encore que la nappe peut être menacée par une baisse considérable si l'on atteint un seuil d'exploitation de $60.10^{3} \mathrm{~m}^{3} / \mathrm{an}$ à travers 75 puits et même par l'intrusion franche et immédiate d'eau marine à partir d'un taux d'exploitation de $180.10^{3} \mathrm{~m}^{3} /$ an (création de 45 nouveaux puits par an).

\section{VI $\square$ CONCLUSION}

La nappe de Chott Maria, à l'instar des nappes urbaines côtières du Sahel tunisien est fragile et tributaire des périodes de pénurie. Si les récentes pluies ont pu voiler, temporairement, les aires de grande salinité, elles n'ont pas pu remédier aux perturbations hydrodynamiques chroniques. La simulation numérique révèle des zones vulnérables qui prennent place directement en aval des aires de pompage (le secteur amont central de la nappe).

En se basant sur nos simulations prévisionnelles, nous recommandons, dans un contexte qui prévoit une pénurie (pluviométrie annuelle de $180 \mathrm{~mm}$ ) durant les cinq prochaines années et une évolution annuelle linéaire des rejets domestiques dans la nappe de $9 \%$, une exploitation à additionner au taux actuel qui ne dépasse pas les $60.10^{3} \mathrm{~m}^{3} / \mathrm{an}$ $\left(7000 \mathrm{~m}^{3} / \mathrm{an} /\right.$ puits à partir de 23 puits). Au delà de ce taux d'exploitation, le risque d'une inversion du sens d'écoulement mer-continent à partir de l'an 2001 est très sérieux [18, 19]. La menace d'une intrusion locale d'eau marine est possible mais un scénario de condamnation de la nappe en la transformant en "dépotoir" de déchets liquides suite à une pollution irréversible, à l'image de la nappe urbaine de Sfax, n'est pas à écarter $[1,2]$. La dégradation de la qualité des eaux, suite à une intrusion marine, pousserait d'avantage les agriculteurs à vendre leurs terres aux promoteurs en immobilier, en industrie et en tourisme. La sauvegarde de la nappe étant encore possible, une gestion qui tienne compte de la multitude des intervenants et surtout de la célérité des changements s'impose. Un suivi plus dense et plus fréquent de la nappe est indispensable et en permettrait un meilleur contrôle, notamment par les simulations numériques.

\section{BIBLIOGRAPHIE}

[1] BEN DHIA H. (1996) - Les nappes phréatiques urbaines: état de l'art et perspectives de gestion (cas de la nappe de Sfax), Sem. APNES, Sfax.

[2] Beni AKHy R., Ben Dhia H., Suissi A., Zammouri M. (1995) - Evolution et modélisation de la nappe phréatique urbaine de Sfax (Tunisie): Relation urbanisation/ eau souterraine. Annales Maghrébines de l'ingénieur; Vol. 9, No.2, pp.25-50.

[3] Spann L. (1996) - Vers une crise de l'eau en Afrique du Nord et au Moyen Orient, La Houille Blanche, No.4, p.10

[4] Detay M., Bersillon J.-L. (1996) - La réalimentation artificielle des nappes profondes: faisabilité et conséquences, La Houille Blanche. No. 4 , p.57-61

[5] Múller O., Detay M. (1993) - Modélisation du comportement des éléments azotés en aquifere alluvial : Importance de l'interface surface-nappe, Hydrogéologie, No. 19, p.1-3.

[6] CRDA. (1995) - Aménagement de l'Oued Nebhana, Rap. int., Sousse.

[7] ONAS (1995) - Assainissement Sousse Nord, Rap. int. Sousse.

[8] SHOEler H. (1962)- Les eaux souterraines, ed Masson et cie., 642p. Paris.

[9] M. Mc. Donal.D \& Patners ltd. (1990) - Etablissement et mise au point du modèle de gestion des nappes du bassin de Goulmima-Errachidia-Boudenib, modèle hydraulique, Rap. 250 p., Cambridge.

[10]DRE Sousse (1996) - La nappe de Chott Maria, Rap. int., CRDA Sousse.

[11] HUBERT C. (1969) - Note sur l'étude hydrogéologique préliminaire des coopératives de Zaarna-Est, Akouda, Hammam Sousse, Sousse Nord et Sousse Sud, Rap. int., CRDA Sousse.

[12]DRE Sousse (1984) - La nappe de Chott Maria, Rap. int. CRDA Sousse.

[13] Lebas Y., Thomas L. (1996)- Analyse des risques et dispositif de suivi: Le cas de d'Izaute et lussagnet, La Houille Blanche, No. 4, pp. 66-71.

[14]Freeze R.-A. \& Cherry J.-A. (1979) - Groundwater, ed. PrenticeHall. Inc., 604p. New Jersey.

[15] SEILLER K.-P. \& LiNDNER W. (1995) - Near surface and deep groundwaters, J. Hydr., No. 165, p.3-44, Amsterdam.

[16] Besbes M., Jerraya F. ET ZAMmouri M. (1991) - MULTIC: simulation des aquifères multicouches en mailles carrés régulières, Rap. ENIT, 13 p., Tunis.

[17] Grove D.-B. \& Stollenwerk K.G. (1984)- Computer model of one dimensional equilibrium controlled sorption processes, US Geological Survey, Water Resources Investigations Report, 84-4059, 58p.

[18] DetaY M., Coll.in J. (1995)- Introduction à la gestion active des aquiferes: concept et philosophie, Hydrogéologie, No.1, p.3-11.

[19] Goodman A. S. (1984)- Principles of water resources planning, ed. Prentice Hall, 563p., New Jersey. 